\title{
Photodynamic therapy for head and neck tumors
}

\author{
Pei-Jen Lou \\ Department of Otolaryngology, National Taiwan University Hospital \\ 7, Chung-Shan South Road, Taipei, Taiwan
}

Tel: 886-2-23123456 ext 5224, Fax: 886-2-23410905, E-mail: pjlou@ha.mc.ntu.edu.tw

\begin{abstract}
Studies in the past have confirmed the safety and efficacy of photodynamic therapy (PDT) in the treatment of various human cancers. Because of the concern for cosmetic and functional preservation, PDT seems to be particularly suited for head and neck tumor treatment. Results from previous studies are promising. Good cosmetic and functional outcomes can be achieved without compromising the patients'survival. PDT is a therapeutic option that may prove a useful addition to the armamentarium of the integrated head and neck oncology team.
\end{abstract}

Because of the complex regional anatomy, head and neck cancers constitute a unique category of human malignancy. Every year, there are about 363,100 new cases of head and neck cancers over the world. Traditional treatment modalities carry significant functional and aesthetic impairment leading to withdrawal and social isolation. The management of head and neck cancers is further complicated by the occurrence of multiple primary malignancies. Treatment of a second primary cancer within the previously operated or irradiated field is even more difficult. The difficulties associated with treating these tumors suggest a need for alternative treatments that are less destructive, repeatable, and compatible with previous and subsequent radiotherapy and surgery.

Photodynamic therapy (PDT) is a non-thermal reaction, the necrosis is localized and healing takes place with little scarring, and good preservation of function. Surgery, radiotherapy, or chemotherapy does not preclude the use of PDT, nor will PDT compromise the subsequent use of any other treatment. PDT is repeatable without cumulative tissue toxicity. Because of these characteristics, PDT is an ideal option in the treatment of head and neck tumors.

PDT has been implemented in the clinical treatment of head and neck tumors since about 20 years ago[1]. In general, patients with early-stage cancers or early recurrences tend to have a very good response to PDT. Complications such as skin phototoxicity are more frequently encountered in the first-generation photosensitizers. Severe adverse events such as carotid blow-out has ever been reported but is very uncommon[2]. 


\section{TH1A-SS-4}

Although early-stage superficial tumors are cured effectively, the results of treating large bulky tumors are disappointing[3]. To overcome the limitations, interstitial PDT has been used to improve the light delivery in tissues. Good treatment responses were obtained and long-term survivors were occasionally encountered[2]. Recent developments in biochemical and molecular technology have improved the specificity and efficacy of photosensitizers. PDT is a therapeutic option that may prove a useful addition to the armamentarium of the integrated head and neck oncology team.

\section{REFERENCES}

[1] P.J.Lou, L.Jones, and C.Hopper. Clinical outcomes of photodynamic therapy for head-and-neck cancer. Technology in Cancer Research \& Treatment 2, 311-317 (2003)

[2] P.J.Lou, H.R.Jager, L.Jones, T.Theodossy, S.G.Bown, and C.Hopper. Interstitial photodynamic therapy as salvage treatment for recurrent head and neck cancer. British Journal of Cancer 91, 441-446 (2004)

[3] C.Hopper, A.Kubler, H.Lewis, I.BingTan, and G.Putnam. mTHPC-mediated photodynamic therapy for early oral squamous cell carcinoma. International Journal of Cancer 111, 138-146 (2004) 